\title{
Expressing the Thermoanaerobacterium saccharolyticum pforA in engineered Clostridium thermocellum improves ethanol production
}

Shuen Hon ${ }^{1,2,3}$, Evert K. Holwerda ${ }^{1,2,3}$, Robert S. Worthen ${ }^{1,2,3}$, Marybeth I. Maloney ${ }^{1,2,3}$, Liang Tian ${ }^{1,2,3}$, Jingxuan Cui ${ }^{2,3,4}$, Paul P. Lin ${ }^{2,5}$, Lee R. Lynd ${ }^{1,2,3,4^{*}}$ and Daniel G. Olson ${ }^{1,2,3^{*}}$

\begin{abstract}
Background: Clostridium thermocellum has been the subject of multiple metabolic engineering strategies to improve its ability to ferment cellulose to ethanol, with varying degrees of success. For ethanol production in C. thermocellum, the conversion of pyruvate to acetyl-CoA is catalyzed primarily by the pyruvate ferredoxin oxidoreductase (PFOR) pathway. Thermoanaerobacterium saccharolyticum, which was previously engineered to produce ethanol of high yield (> 80\%) and titer ( $70 \mathrm{~g} / \mathrm{L})$, also uses a pyruvate ferredoxin oxidoreductase, $p f o r A$, for ethanol production.

Results: Here, we introduced the T. saccharolyticum pforA and ferredoxin into C. thermocellum. The introduction of pforA resulted in significant improvements to ethanol yield and titer in C. thermocellum grown on $50 \mathrm{~g} / \mathrm{L}$ of cellobiose, but only when four other T. saccharolyticum genes (adhA, $n f n A, n f n B$, and adh $E^{G 544 D}$ ) were also present. T. saccharolyticum ferredoxin did not have any observable impact on ethanol production. The improvement to ethanol production was sustained even when all annotated native C. thermocellum pfor genes were deleted. On high cellulose concentrations, the maximum ethanol titer achieved by this engineered C. thermocellum strain from $100 \mathrm{~g} / \mathrm{L}$ Avicel was $25 \mathrm{~g} / \mathrm{L}$, compared to $22 \mathrm{~g} / \mathrm{L}$ for the reference strain, LL1319 (adhA(TSC)-nfnAB(TSC)-adh $E^{\mathrm{GS} 44 \mathrm{D}}$ (TsC)) under similar conditions. In addition, we also observed that deletion of the C. thermocellum pfor 4 results in a significant decrease in isobutanol production.
\end{abstract}

Conclusions: Here, we demonstrate that the pforA gene can improve ethanol production in C. thermocellum as part of the T. saccharolyticum pyruvate-to-ethanol pathway. In our previous strain, high-yield ( $75 \%$ of theoretical) ethanol production could be achieved with at most $20 \mathrm{~g} / \mathrm{L}$ substrate. In this strain, high-yield ethanol production can be achieved up to $50 \mathrm{~g} / \mathrm{L}$ substrate. Furthermore, the introduction of pforA increased the maximum titer by $14 \%$.

Keywords: Consolidated bioprocessing, Clostridium thermocellum, Thermoanaerobacterium saccharolyticum, Pyruvate ferredoxin oxidoreductase, Ethanol, Isobutanol

*Correspondence: Lee.R.Lynd@dartmouth.edu;

Daniel.G.Olson@dartmouth.edu

${ }^{1}$ Thayer School of Engineering, Dartmouth College, 14 Engineering Drive,

Hanover, NH 03755, USA

Full list of author information is available at the end of the article 


\section{Background}

Clostridium thermocellum is a promising candidate organism for the consolidated bioprocessing (CBP) of lignocellulosic biomass into biofuels, such as ethanol [1]. Metabolic engineering of $C$. thermocellum has improved ethanol yields and titers; however, further improvements are needed for commercial viability [1-3]. The metabolic engineering strategies pursued in $C$. thermocellum have encompassed restricting native metabolism toward ethanol production, as well as heterologous expression of ethanol production pathways in C. thermocellum; the improvements to ethanol yield and titer of these various approaches have been previously summarized [4].

Recently, four proteins from a strain of Thermoanaerobacterium saccharolyticum engineered for high levels of ethanol production (strain M1442, Herring et al. [5])-namely, an NADPH-dependent alcohol dehydrogenase (AdhA), the NADH-dependent reduced ferredoxin:NADP+ oxidoreductase complex (NfnAB), and a mutant bifunctional alcohol dehydrogenase (AdhE ${ }^{\mathrm{G} 544 \mathrm{D}}$ ) - were introduced into wild-type C. thermocellum, to improve ethanol yield, titer, and production rate [4]. However, the maximum ethanol titer achieved by this engineered $C$. thermocellum strain (LL1319) was only $15 \mathrm{~g} / \mathrm{L}$, which is far short of the $70 \mathrm{~g} / \mathrm{L}$ ethanol titer that engineered T. saccharolyticum (strain M1442) is capable of producing [5].

In both $C$. thermocellum and $T$. saccharolyticum, the oxidative decarboxylation of pyruvate to acetylCoA is primarily catalyzed by a pyruvate ferredoxin oxidoreductase (PFOR) enzyme or enzyme complex [6-9]. In C. thermocellum, there are five candidates (Table 1) [10]; of these, the pfors encoded by the genes Clo1313_0020-0023 and Clo1313_1353-1356 were reported to be cumulatively responsible for approximately $80 \%$ of the PFOR activity [10]. However, it is not known which of these five pfors is important for ethanol production. In addition, prior to this work, all strains of C. thermocellum that had been engineered to produce ethanol with the pfor pathway have relied on the native pfors; among these strains, the maximum ethanol titers

Table 1 Gene names and locus numbers for five annotated C. thermocellum pfor genes or gene clusters

\begin{tabular}{ll}
\hline pfor name & DSM 1313 locus identifiers \\
\hline pfor1 & Clo1313_0020-0023 \\
pfor2 & Clo1313_0382-0385 \\
pfor3 & Clo1313_0673 \\
pfor4 & Clo1313_1353-1356 \\
pfor5 & Clo1313_1615-1616 \\
\hline
\end{tabular}

observed were around $25 \mathrm{~g} / \mathrm{L}$, suggesting therefore that the native pfors may not be capable of supporting ethanol production beyond that titer. By comparison in $T$. saccharolyticum, there are six genes annotated as putative pfors, and deletion studies have shown that the pforA gene (Tsac_0046 in strain DSM 8691) encodes the primary Pfor protein in T. saccharolyticum, as evidenced by the significant decrease in PFOR activity and ethanol production in strains which had the pforA gene deleted [11]. These results suggested that the pfor $A$, unlike the $C$. thermocellum pfors, was capable of supporting ethanol production to high titers $(>40 \mathrm{~g} / \mathrm{L})$.

Another important component of the pyruvate to ethanol pathway that utilizes a Pfor enzyme is ferredoxin, which is responsible for electron transfer from pyruvate to nicotinamide cofactors, in the context of ethanol production [12]. Previous work suggests that C. thermocellum ferredoxins are compatible with $T$. saccharolyticum ferredoxin-utilizing enzymes [13]. However, given that they play an important role in electron transfer, and that it is possible that some ferredoxins work better than others with certain enzymes, we decided to include the introduction of T. saccharolyticum ferredoxin into C. thermocellum in this study. We chose the Tsac_2084 ferredoxin because of its location adjacent to $a d h A$ and $n f n A B$ (Tsac_2087 and Tsac_2086-2085, respectively) on the chromosome; given that these latter two enzymes were previously shown to be important for ethanol production, both in T. saccharolyticum $[14,15]$ and C. thermocellum [4], this ferredoxin seemed to be a reasonable choice.

In this study, we hypothesized that introducing the $T$. saccharolyticum pforA would improve ethanol production in a strain of $C$. thermocellum that had been previously engineered with other $T$. saccharolyticum ethanol production enzymes (i.e., strain LL1319). We also investigated whether the introduced PforA protein would be able to sustain ethanol production after the five known C. thermocellum Pfor enzyme and enzyme complexes were deleted (Fig 1). We also sought to determine the importance of T. saccharolyticum ferredoxin for ethanol production. We tested this by integrating T. saccharolyticum pforA (with or without the ferredoxin Tsac_2084) into C. thermocellum strains, and evaluating the effects that these genes had on enzyme activity, and on ethanol yield, titer, and maximum ethanol production rate, on soluble carbon sources as well as high loadings of crystalline cellulose.

\section{Methods}

Strain and plasmid construction

Table 2 lists all strains used and constructed in this study, and the plasmids used for integrating and deleting genes 
of interests. All plasmids were constructed via isothermal assembly [16] using a commercial kit sold by New England Biolabs (NEBuilder ${ }^{\circledR}$ HiFi DNA Assembly Master Mix, catalog number E2621). Purification of plasmid DNA or PCR products for cloning was done using commercially available kits from Qiagen, Zymo Research, or New England Biolabs. C. thermocellum strains were transformed using previously described methods [17]. Plasmid DNA destined for transformation into C. thermocellum was purified from Escherichia coli BL21 derivative strains (New England Biolabs catalog number C2566) to ensure that the DNA was properly methylated [18].

\section{Media preparation and culture conditions}

All reagents used in this study were of molecular grade and obtained from either Sigma Aldrich or Fisher Scientific, unless otherwise noted. C. thermocellum strains were grown at $55^{\circ} \mathrm{C}$ under anaerobic conditions, either in conical tubes in an anaerobic chamber (Coy Laboratory Products, Grass Lakes, MI), with previously described environmental conditions [4], or in sealed $150 \mathrm{~mL}$ serum bottles that were prepared and inoculated as previously described [4].

Complex medium (CTFUD) was prepared as previously described and used to culture C. thermocellum cells in preparation for transformations, or for harvesting genomic DNA for strain resequencing.

Table 2 List of strains and integration/deletion plasmids used in this study

\begin{tabular}{|c|c|c|c|c|}
\hline Strains/plasmids & Organism & Description & Accession number & References or source \\
\hline E. coli T7 express & Escherichia coli & $\begin{array}{l}\text { fhuA2 lacZ::T7 gene1 [lon] ompT gal sulA11 R(mer- } \\
\text { 73:::miniTn10-TetS)2 [dcm] R(zgb-210::Tn10-TetS) } \\
\text { endA1 } \Delta \text { (mcrC-mrr) 114::IS10 }\end{array}$ & & New England Biolabs (Ipswich, MA) \\
\hline M1442 & T. saccharolyticum & Engineered and evolved T. saccharolyticum & SRA233073 & {$[5]$} \\
\hline LL1004 & C. thermocellum & DSM 1313 & СР002416 & DSMZ \\
\hline AG929 & C. thermocellum & DSM1313 $\Delta h p t \Delta c l 01313 \_0478$ & SRP097241 & {$[4]$} \\
\hline LL1319 & C. thermocellum & $\begin{array}{l}\text { AG929 P Plo1313_2638::adhA(TSC)-nfnAB(TSC)-adh } E^{G 544 D} \\
(\text { TSC) }\end{array}$ & SRP101300 & {$[4]$} \\
\hline LL1565 & C. thermocellum & AG929 Clo1313_2637::P pforA(Tsc) pforA(Tsc) -fd(Tsc) & SRP144031 & This study \\
\hline LL1391 & C. thermocellum & LL1319 Clo1313_2637:: Pfora(Tsc) pforA(Tsc)-fd(Tsc) & SRP141156 & This study \\
\hline LL1566 & C. thermocellum & LL1319 Clo1313_2637::P pfora(Tsc) pforA(Tsc) & SRP144035 & This study \\
\hline LL1436 & C. thermocellum & LL1391 هClo1313_0020-0023 & SRP144013 & This study \\
\hline LL1437 & C. thermocellum & LL1391 $\Delta$ Clo1313_1353-1356 & SRP144038 & This study \\
\hline LL1438 & C. thermocellum & LL1436 $\Delta$ Clo1313_1353-1356 & SRP144037 & This study \\
\hline LL1567 & C. thermocellum & LL1437 $\Delta$ Clo1313_0020-0023 & SRP144045 & This study \\
\hline LL1568 & C. thermocellum & LL1438 $\Delta$ Clo1313_0673 & SRP144054 & This study \\
\hline LL1569 & C. thermocellum & LL1568 $\Delta$ Clo1313_0382-0385 & SRP144051 & This study \\
\hline LL1570 & C. thermocellum & LL1569 $\triangle$ Clo1313_1615-1616 & SRP144049 & This study \\
\hline LL1556 & C. thermocellum & 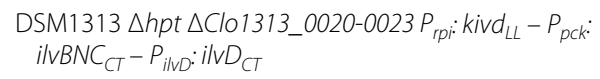 & SRP144036 & This study \\
\hline LL1559 & C. thermocellum & DSM1313 $\Delta h p t \Delta C l 01313 \_0382-0385$ & SRP144040 & This study \\
\hline LL1563 & C. thermocellum & DSM1313 $\Delta$ hpt $\triangle$ Clo1313_0673 & & This study \\
\hline LL1564 & C. thermocellum & DSM1313 Ahpt $\Delta$ Clo1313_1353-1356 & & This study \\
\hline LL1560 & C. thermocellum & DSM1313 Ahpt $\Delta$ Clo1313_1615-1616 & SRP144039 & This study \\
\hline pJGW37 & & C. thermocellum expression plasmid & & [19] \\
\hline pSH105 & & $P_{\text {enolase }}$ PforA $(T s c)-f d(T s c)$ integration vector & $\mathrm{MH} 245114$ & This study \\
\hline pSH106 & & $P_{\text {pforA }(T s c)} p f o r A(T s C)-f d(T s c)$ integration vector & $\mathrm{MH} 245115$ & This study \\
\hline pSH107 & & $P_{\text {Athe_2105 }}$ Pfor $A(T S C)-f d(T S C)$ integration vector & $\mathrm{MH} 245116$ & This study \\
\hline pSH121 & & $P_{\text {pforA(TsC) }}$ pforA(TsC) integration vector & MH245113 & This study \\
\hline pDGO77 & & Clo1313_0020-0023 deletion vector & $\mathrm{MH} 245117$ & This study \\
\hline pDGO78 & & Clo1313_0673 deletion vector & MH245118 & This study \\
\hline pSH116 & & Clo1313_1353-1356 deletion vector & $\mathrm{MH} 245112$ & This study \\
\hline pSH130 & & Clo1313_0382-0385 deletion vector & $\mathrm{MH} 245110$ & This study \\
\hline pSH131 & & Clo1313_1615-1616 deletion vector & $\mathrm{MH} 245111$ & This study \\
\hline
\end{tabular}


The defined medium (MTC-5) was prepared as previously described [4] and used for all other purposes. Cellobiose was used as the main carbon source, unless otherwise noted. For MTC-5, $5 \mathrm{~g} / \mathrm{L}$ cellobiose was used for routine culture and growing cells for gene expression analyses (see "Measuring gene expression") or enzyme assays (see "Enzyme assays"). Cellobiose concentrations of $20 \mathrm{~g} / \mathrm{L}$ and $50 \mathrm{~g} / \mathrm{L}$ were used for fermentation end product analyses; when $50 \mathrm{~g} / \mathrm{L}$ cellobiose was used, the concentrations of pyridoxamine dihydrochloride, P-aminobenzoic acid, D-biotin, and vitamin B12 used were doubled to a final concentration of $0.04 \mathrm{~g} / \mathrm{L}$, $0.008 \mathrm{~g} / \mathrm{L}, 0.008 \mathrm{~g} / \mathrm{L}$, and $0.004 \mathrm{~g} / \mathrm{L}$, respectively.

Specific growth rates, measured on MTC- 5 medium with $5 \mathrm{~g} / \mathrm{L}$ cellobiose as the main carbon source and using a microplate reader, were determined as previously described [20].

\section{Measuring gene expression}

Gene expression was determined using reverse transcription quantitative PCR (RT-qPCR); Additional file 1: Table S1 lists the primers used for RT-qPCR. Cultures were grown on MTC-5 medium to mid-exponential phase $\left(\mathrm{OD}_{600}\right.$ between 0.6 and 1.0); cultures were then processed as previously described [4]. Gene expression was normalized against $C$. thermocellum recA expression [21] to allow comparison across strains, as was previously done [4, 22, 23].

Translation initiation efficiencies were calculated using an online calculator from the Howard M. Salis group website (URL: https://salis.psu.edu/software/reverse) [24, 25].

\section{Enzyme assays}

Cell cultures were grown, harvested, stored, and lysed to obtain cell-free extract as previously described [4]. Protein concentrations in the cell-free extracts were determined using Bio-Rad (Hercules, CA) protein dye assay reagent, with bovine serum albumin used as a protein standard.

All enzyme assays were performed at $55^{\circ} \mathrm{C}$ and at $\mathrm{pH}$ 7.0 under anaerobic conditions in an anaerobic chamber (Coy Laboratory Products, Grass Lakes, MI).

Pyruvate ferredoxin oxidoreductase (PFOR) activity was assayed by the reduction of benzyl viologen instead of methyl viologen, a modification of a previously described protocol [11]. The reduction of benzyl viologen was monitored at a wavelength of $578 \mathrm{~nm}$, and an extinction coefficient of $7.8 \mathrm{mM}^{-1} \mathrm{~cm}^{-1}$ was used to calculate activity [26]. The assay mixture contained $100 \mathrm{mM}$ Tris- $\mathrm{HCl}$, $5 \mu \mathrm{M} \mathrm{FeSO}_{4}, 0.5 \mathrm{mM}$ DTT, $2 \mathrm{mM} \mathrm{MgCl}, 0.4 \mathrm{mM}$ coenzyme A, $0.4 \mathrm{mM}$ thiamine pyrophosphate, and $1 \mathrm{mM}$ benzyl viologen dichloride. Cell extract was added to this assay mixture first to establish a baseline; the reaction was then started by adding $2 \mathrm{mM}$ sodium pyruvate. The final volume for all biochemical assays was $1200 \mu \mathrm{L}$.

\section{High solids fermentations}

Bioreactor experiments were carried out as previously described [27], with an initial working volume of $1 \mathrm{~L}$. MTC-5 medium [4] was modified to have $5 \mathrm{~g} / \mathrm{L}$ initial urea instead of $2 \mathrm{~g} / \mathrm{L}$ as previously reported, Vitamin and trace mineral concentrations were increased to $4 \times$ and $5 \times$ of previously reported values [4]. $100 \mathrm{~g} / \mathrm{L}$ crystalline cellulose (Avicel PH105) was used as the main carbon source. Bioreactors were inoculated with $5 \mathrm{~mL}$ of an overnight culture $(0.5 \% \mathrm{v} / \mathrm{v}$ inoculum $)$ that was grown on MTC- 5 medium modified to contain $5 \mathrm{~g} / \mathrm{L}$ MOPS sodium salt and to use $5 \mathrm{~g} / \mathrm{L}$ Avicel as the main carbon source. $\mathrm{pH}$ was maintained at $7.00 \pm 0.05$ by the automatic addition of $4 \mathrm{~N} \mathrm{KOH}$.

\section{Analytical methods}

The fermentation products were measured by highpressure liquid chromatography (HPLC) as previously described [27]. For tube and serum bottle cultures, the results were normalized against an internal standard (MOPS buffer) to account for variation due to sample processing and handling. Headspace gas composition for serum bottle fermentations was measured as previously described [23].

To quantify extracellular amino acids, samples were first derivatized with a commercially available derivatization reagent (Accq-Tag Chemistry kit, catalog number WAT052875, Waters Corporation, Milford, MA). The derivatized samples were run on an HPLC equipped with the Waters AccQ.Tag column (part number WAT052885, Waters Corporation, Milford, MA) with fluorescence detection, using an excitation wavelength of $250 \mathrm{~nm}$ and emission wavelength of $395 \mathrm{~nm}$, following the manufacturer's recommended instrument method. Sample preparation was performed according to the manufacturer's instructions (manual number WAT052874, Rev 1, Waters Corporation, Milford MA).

Residual cellulose (Avicel PH105) concentration was determined via quantitative saccharification [28]. Pellet nitrogen (a proxy for cell biomass) was measured with a Shimadzu TOC-V CPH elemental analyzer with TNM-1 and ASI-V modules (Shimadzu Corp., Columbia, MD), as previously described $[28,29]$.

Volumetric ethanol production from bioreactor experiments was determined by fitting ethanol production data points for each fermentation with the five-parameter sigmoidal Richards equation [30], and 
calculating the first derivative of each fermentation's fitted Richards equation.

\section{Sequencing and resequencing}

Routine Sanger sequencing of plasmids was performed by Genewiz. Inc., with at least twofold coverage of the cloned regions. Whole genome resequencing of strains was performed by the Department of Energy Joint Genome Institute, using the Illumina Miseq sequencing platform, with an average of 100-fold coverage. Sequencing data were analyzed with the CLC Genomics workbench, using strain LL1319 as the reference genome (accession number SRP101300). Accession numbers for strains and plasmids are listed in Table 2. A summary of the resequencing results can be found in Additional file 2.

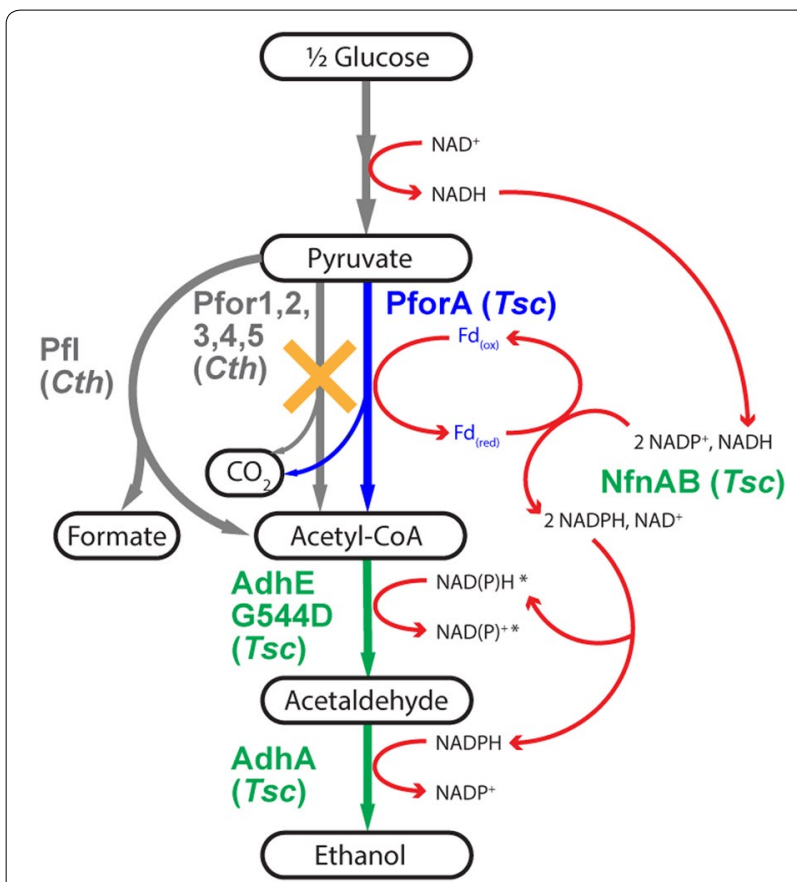

Fig. 1 Pyruvate to ethanol production in C. thermocellum using the T. saccharolyticum pyruvate to ethanol pathway; this figure is adapted from Hon et al. [4]. Metabolites and products are colored black. Native genes ( $C$ th) and the pathways they correspondingly catalyze are colored gray; T. saccharolyticum genes (Tsc) that were previously introduced in Hon et al. [4], as well as the reactions that they catalyze, are colored green. Red arrows represent electron flux. The T. saccharolyticum pforA and ferredoxin introduced in this study are depicted in blue. The orange cross represents the pathways that were deleted in this study

\section{Results}

Choosing a promoter to drive pforA expression

Thermoanaerobacterium saccharolyticum pforA and ferredoxin were integrated into the $C$. thermocellum genome in strain LL1319, at a location immediately downstream of Clo1313_2637, and before the putative promoter for the Clo1313_2635 gene (Additional file 1: Figure S1). This was done so as to locate the new operon close to the previously introduced $T$. saccharolyticum adhA-nfnAB-adhE $E^{G 54 D}$ operon [4], without disrupting a putative peroxiredoxin two-gene cluster (Clo1313_26382637). Three promoters were tested to drive expression of the pfor $A$-ferredoxin operon: the previously described $C$. thermocellum enolase promoter [31], the T. saccharolyticum pforA promoter, and the Athe_2105 promoter from Caldicellulosiruptor bescii [19] with a modified ribosome binding site [24]. Promoter sequences and predicted translation initiation efficiencies of $p$ for $A$ are reported in Additional file 1: Table S2.

T. saccharolyticum pfor $A$ and ferredoxin expression was observed in all three operon configurations (Fig. 2a), but not in wild-type $C$. thermocellum and the parent strain, LL1319, as expected. In general, we observed that ferredoxin expression was lower than that of the pforA gene. It was also observed that the $C$. thermocellum enolase promoter resulted in the highest level of gene expression, followed by the T. saccharolyticum pfor A promoter, with the modified Athe_2105 promoter giving the lowest level of expression. However, we also observed that expression was more variable with the $C$. thermocellum enolase promoter than with the two heterologous promoters. The variation observed with the native enolase promoter is similar to what we have previously observed for lacZ expression [31].

Fermentation product profiles for the C. thermocellum strains using the native enolase and T. saccharolyticum pfor $A$ promoters were indistinguishable from the parent strain LL1319 on $20 \mathrm{~g} / \mathrm{L}$ initial cellobiose. The strain that used the C. bescii Athe_2105 promoter, however, showed an unexpected decrease in ethanol production (Fig. 2b). On 52 g/L initial cellobiose, we again observed that the Athe_2105 promoter-containing strain exhibited reduced ethanol production compared to the parent strain LL1319 (Fig. 2c), unlike the other two strains that contained the $T$. saccharolyticum pfor $A$ and ferredoxin; this strain was thus excluded from further investigations. Between the two other strains, both showed comparable improvements to ethanol production over the parent strain LL1319, with the T. saccharolyticum pforA promoter-driven strain showing slightly higher ethanol titers. Given that the T. saccharolyticum pfor $A$ promoter resulted in the highest levels of ethanol production of the three promoters tested, and that its use 


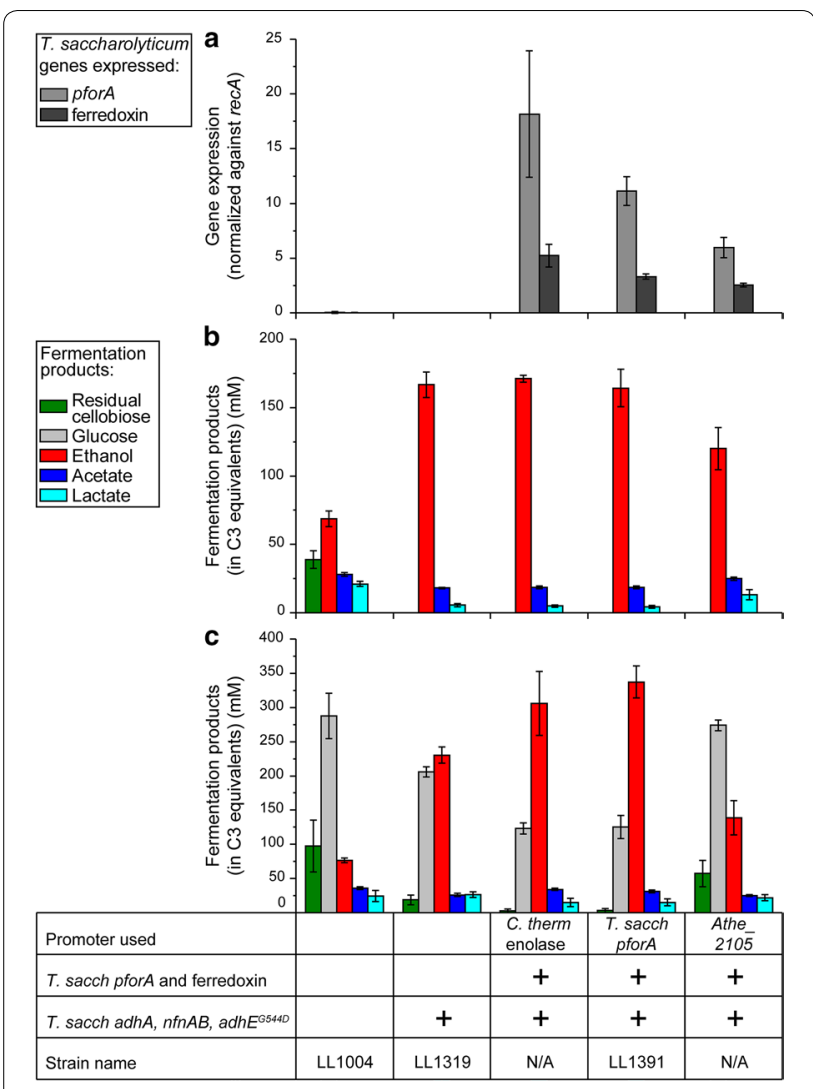

Fig. 2 a Relative expression levels (normalized against $C$. thermocellum recA expression) of T. saccharolyticum pforA and ferredoxin. $\mathbf{b}, \mathbf{c}$ Fermentation products of five $C$. thermocellum strains. Cultures were grown in $15 \mathrm{~mL}$ tubes with $5 \mathrm{~mL}$ of MTC-5 medium, with $60 \pm 2 \mathrm{mM}(\sim 20 \mathrm{~g} / \mathrm{L})(\mathbf{b})$ or $151 \pm 3 \mathrm{mM}(\sim 52 \mathrm{~g} / \mathrm{L})(\mathbf{c})$ initial cellobiose, for $72 \mathrm{~h}$ and $168 \mathrm{~h}$, respectively, at $55^{\circ} \mathrm{C}$. Error bars represent one standard deviation ( $n=3$ for $\mathbf{a}$ and $\mathbf{b}, n=5$ for $\mathbf{c}$ )

avoids duplicating native DNA sequences (which can lead to unintended recombination events and complicate the analysis of resequencing data), we proceeded forward with this strain and designated it as strain LL1391 (Table 2). Subsequent fermentations confirmed that strain LL1391 produced more ethanol than strain LL1319 (Fig. 3b), which may be attributed to a significant increase in the BV:PFOR specific enzyme activity in strain LL1391 (unpaired two-tailed $t$ test, $\mathrm{p}=0.0045$ ) (Fig. 3a).

\section{The effects of $T$. saccharolyticum pforA and ferredoxin expression on ethanol production}

Strain LL1319 contains four T. saccharolyticum genes from previous strain development [4]; we therefore investigated whether the improvements in ethanol production were dependent on the presence of the previously introduced T. saccharolyticum adhA, nfnAB, and adh $E^{G 544 D}$ genes. Strain LL1565 was created by integrating $T$. saccharolyticum pforA and ferredoxin, driven by the T. saccharolyticum pforA promoter (plasmid pSH106, Table 2) into strain AG929 (the parent strain of strain LL1319). A significant increase in BV:PFOR specific activity in strain LL1565 was observed, relative to strain AG929 (unpaired two-tailed t test, $\mathrm{p}=0.0064$ ); the measured specific activity of strain LL1565 was comparable to activity levels measured in strain LL1391 (Fig. 3a), indicating that the introduced T. saccharolyticum PforA protein was present and active in the strain. Ethanol production with strain LL1565 appeared to be slightly improved relative to parent strain AG929, although the metabolic yield and final titers were significantly lower than that of strain LL1319 and by extension that of LL1391 (Fig. 3b), suggesting that the improvement in ethanol production from introducing $p$ for $A$ is dependent on the presence of the other T. saccharolyticum ethanol production pathway genes.

To determine whether the T. saccharolyticum ferredoxin was necessary for the improvements in ethanol production, $T$. saccharolyticum pforA alone was integrated into strain LL1319 at the same locus as was done in strain LL1391 to create strain LL1566 (using plasmid pSH121, see Table 2). The BV:PFOR specific enzyme activity for strain LL1566 was no different from that observed in LL1391, as expected; fermentation products for the two strains were also similar, suggesting that the introduced T. saccharolyticum PforA protein was responsible for the improvements in ethanol production (Fig. 3). We attempted to introduce the T. saccharolyticum ferredoxin on its own into strain LL1319, but were not successful. Given that there appeared to be no detrimental effects in ethanol production due to the presence of $T$. saccharolyticum ferredoxin (Fig. 3), and that the ferredoxin is important in the production of ethanol as an electron carrier, we decided to retain it in subsequent strains (see Table 2 for strain lineage).

\section{The effect of deleting native pfors on ethanol production}

The introduction and expression of $T$. saccharolyticum pforA have thus far been associated with an increase in BV:PFOR specific activities, and an increase in ethanol titer and metabolic yield (Fig. 3). However, the strains evaluated so far still contain the five native Pfor-encoding genes and gene clusters (Table 1). To better determine whether the improvements in ethanol production were due to the introduced T. saccharolyticum PforA protein, we deleted all five $C$. thermocellum pfor gene clusters in an iterative manner.

Previous work suggested that pfor1 (Clo1313_00200023) and pfor4 (Clo1313_1353-1356) encoded for the main Pfor protein complexes in $C$. thermocellum [10]. Further support for pfor 1 and pfor4 encoding for 
important Pfor complexes in C. thermocellum was found when it was observed that BV:PFOR specific activity decreased by $\sim 80 \%$ relative to wild-type $C$. thermocellum (Additional file 1: Figure S2) when either pfor1 or pfor4 was deleted in C. thermocellum (strain LL1556 and LL1564). Strains containing a deletion of either pfor 2 or pfor 5 did not show any significant differences in BV:PFOR specific activity, suggesting that they were not important for PFOR activity in C. thermocellum, or that pyruvate was not the primary substrate for these enzymes. The deletion of pfor 3 , which bears the most similarity to the T. saccharolyticum pfor $A$, also resulted in $\sim 40 \%$ decrease in specific BV:PFOR activity. Given these observations, pfor 1 and pfor4 were therefore the first targets for gene deletion in strain LL1391.
Starting with strain LL1391 (wt strain expressing $T$. saccharolyticum pathway, including pforA), deletion of pfor1 (strain LL1436) did not result in any significant effects on ethanol production or enzyme specific activity. Deletion of pfor4 (strain LL1437), however, showed a decrease in ethanol yield and a large decrease in titer (Fig. 3b), despite very little change in BV:PFOR activity (LL1391 vs. LL1437, unpaired two-tailed $t$ test, $\mathrm{p}=0.23$ ) (Fig. 3a).

Resequencing analyses subsequently revealed that LL1437 contained a 1207G > T mutation in the coding sequence for the Clo1313_1483 gene that resulted in a G403* nonsense mutation in the amino acid sequence; excluding the targeted $p$ for deletions, there were no other differences between the genomes of the two strains.

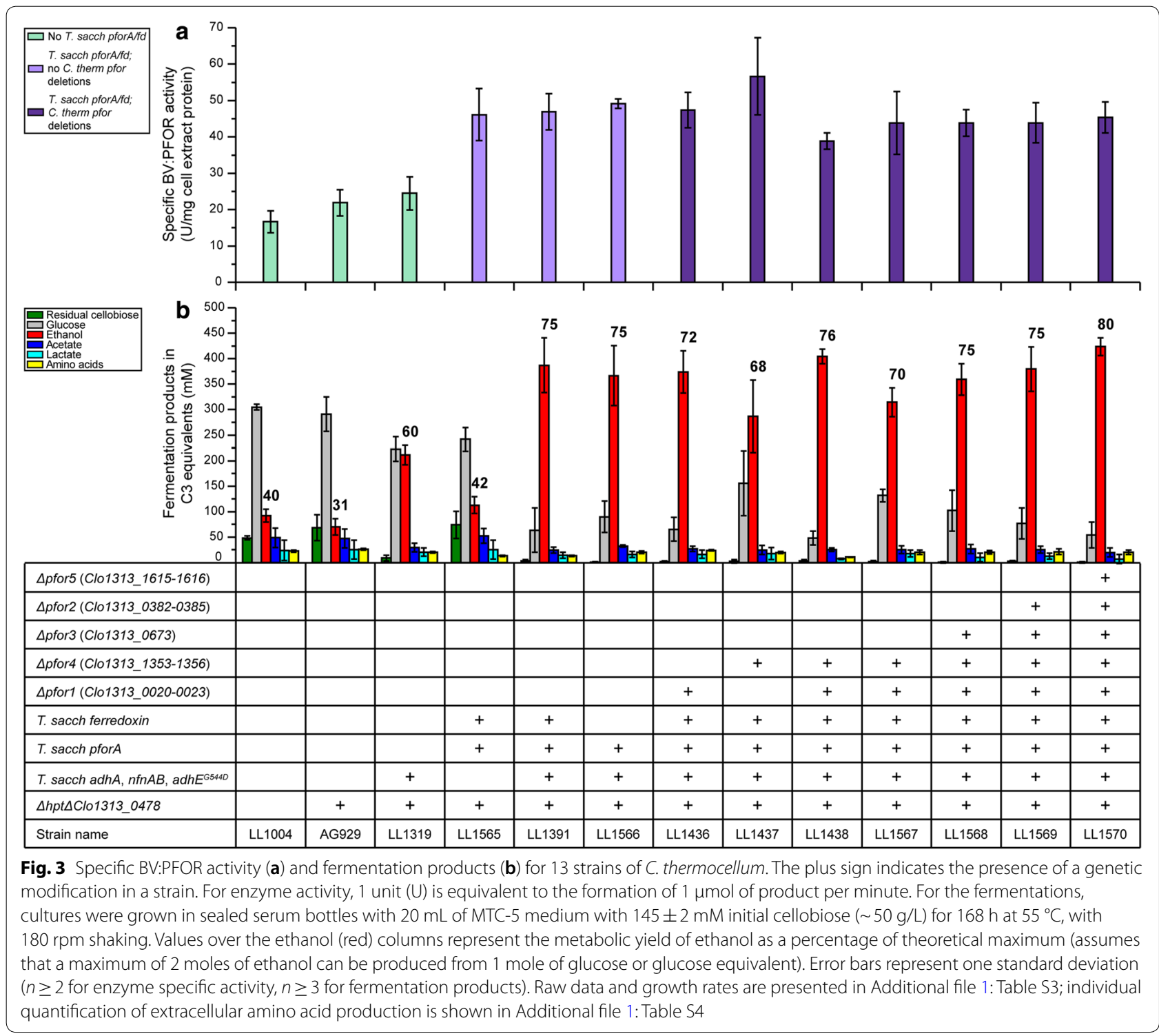


Clo1313_1483 is annotated as encoding a predicted pyrroloquinoline quinone-associated protein, and previous work suggests that it is expressed in C. thermocellum strain ATCC27405 [32, 33]; however, its function in $C$. thermocellum strain DSM1313 is unknown.

To compare the effect of the Clo1313_1483 mutation vs. pfor4 deletion, we constructed two pfor1/pfor4 double deletion strains: LL1438 (LL1436 with pfor4 deleted) and LL1567 (LL1437 with pfor1 deleted). Since neither of these strains showed any significant difference in ethanol production relative to their respective parent strains (LL1438 vs. LL1436 and LL1567 vs. LL1437), the difference in ethanol production between LL1436 and LL1437 is likely due to the Clo1313_1483 mutation, and not the effect of the pfor 4 deletion.

The double pforl/pfor4 deletion strain, LL1438, was still able to sustain the improved ethanol production observed in strain LL1391. To eliminate the possibility that this was due to the remaining three $C$. thermocellum Pfor enzymes compensating for the pfor 1 and pfor 4 deletions, the genes encoding for pfor 3 , pfor 2 , and pfor 5 (see Table 1 for gene numbers) were iteratively deleted to create strain LL1570. Strain LL1570 was able to produce $424 \pm 13 \mathrm{mM}(\sim 20 \mathrm{~g} / \mathrm{L})$ of ethanol from $50 \mathrm{~g} / \mathrm{L}$ of cellobiose, with a metabolic ethanol yield of $80 \%$ of the theoretical maximum (Fig. 4b; also see Additional file 1: Table S3), an improvement over the reference strain LL1319, which was previously reported to have achieved a maximum ethanol yield of $74 \%$ of theoretical maximum on $20 \mathrm{~g} / \mathrm{L}$ cellobiose, and a maximum ethanol titer of $326 \mathrm{mM}(\sim 15 \mathrm{~g} / \mathrm{L})$ on $60 \mathrm{~g} / \mathrm{L}$ Avicel (120). The maximum specific growth rate of strain LL1570 on cellobiose was unaffected relative to the starting strain, LL1319 (Additional file 1: Table S3), suggesting that deleting the native pfor genes did not result in any growth defects, and that the introduced T. saccharolyticum pfor A complemented the deletions of these five native $C$. thermocellum pfor genes.

In strain LL1570, we would have expected that deletion of pforA would eliminate acetate and ethanol production and divert flux to lactate production (similar to what was observed for pfor deletions in T. saccharolyticum [11]). Despite several attempts to delete $p$ for $A$ in this strain, we were not successful. Although not conclusive, this negative result suggests that PFOR activity is essential in this strain, and that pforA is the source of that activity.

\section{Fermentation of high cellulose concentrations}

To determine if replacing of the native pfors with the $T$. saccharolyticum pforA and ferredoxin had improved the maximum ethanol titer and maximum volumetric production rate of ethanol, and to evaluate the performance of the strain on a cellulosic substrate, strains LL1319 and LL1570 were grown on $100 \mathrm{~g} / \mathrm{L}$ Avicel microcrystalline cellulose.

Strains LL1319 and LL1570 consumed about the same amount of Avicel (Figs. 3b and 4a, see also Additional file 1: Table S5), but produced different amounts of ethanol. Strain LL1319 produced ethanol to a titer of $486 \pm 5 \mathrm{mM}(\sim 22 \pm 0.2 \mathrm{~g} / \mathrm{L})$ (Fig. 4c), for a metabolic yield of $45 \%$ of the theoretical maximum; the ethanol titer observed here was higher than previously reported [4], although it should be noted that the media composition was different. In contrast, strain LL1570 produced $551 \pm 32 \mathrm{mM}(25 \pm 1.5 \mathrm{~g} / \mathrm{L})$ of ethanol (Fig. $4 \mathrm{~d}$, Additional file 1: Figure S3), for a metabolic yield of $54 \%$ of theoretical maximum. The results provide further evidence that T. saccharolyticum pfor $A$ improved ethanol yield and titer (unpaired two-tailed $t$ test; $\mathrm{p}=0.003$ for ethanol yield, $\mathrm{p}=0.02$ for ethanol titer). The differences in volumetric productivity of ethanol between strains LL1319 and LL1570 $\left(0.66 \pm 0.03 \mathrm{~g} \mathrm{~L}^{-1} \mathrm{~h}^{-1}\right.$ and $0.70 \pm 0.12 \mathrm{~g} \mathrm{~L}^{-1} \mathrm{~h}^{-1}$, respectively) were not statistically significant (unpaired two-tailed $t$ test; $\mathrm{p}=0.338$ ) (Additional file 1: Table S5). The ethanol titer of $25 \mathrm{~g} / \mathrm{L}$ for strain LL1570 was very similar to that produced by another engineered strain of C. thermocellum, LL1210 ( $\Delta$ hpt $\Delta$ hydG $\Delta l d h \Delta p f l \Delta p t a-$ ack adhE(D494G)), which was generated by eliminating the native competing carbon and electron pathways, followed by strain adaptation over $\sim 2500$ generations to increase growth rate, and which was reported to produce $27 \mathrm{~g} / \mathrm{L}$ of ethanol from $95 \mathrm{~g} / \mathrm{L}$ of Avicel [34]. The byproduct concentrations (organic acids and total extracellular amino acids) in both strains LL1319 and LL1570 were similar, except for isobutanol production, which decreased to below our limit of quantification $(0.1 \mathrm{mM})$ in strain LL1570 (Fig. 4d) (strain LL1319 produced a maximum isobutanol titer of $\sim 14 \mathrm{mM}$; see Fig. $4 \mathrm{c}$ ); this suggests that one of the deleted $C$. thermocellum pfors may be involved in the biosynthesis of isobutanol. Fermentation results from a set of $C$. thermocellum strains that contain a deletion of one of the five annotated pfors suggest that it is pfort that is associated with isobutanol production (Additional file 1: Figure S4).

\section{Discussion and conclusion}

In this work, we investigated the effects of $T$. saccharolyticum pfor $A$ and ferredoxin on ethanol production in $C$. thermocellum. There was no effect from expressing $T$. saccharolyticum ferredoxin in C. thermocellum. It is known that ferredoxins from one organism can often transfer electrons to proteins from another organism [35], so it would not be surprising if one of the native C. thermocellum ferredoxins was sufficient for electron transfer from T. saccharolyticum Pfor protein. 


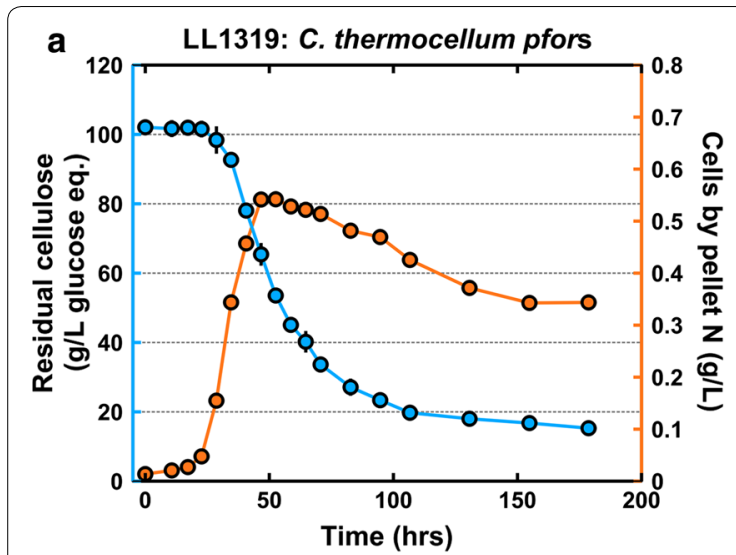

\$. Residual cellulose (g/L glucose eq.)
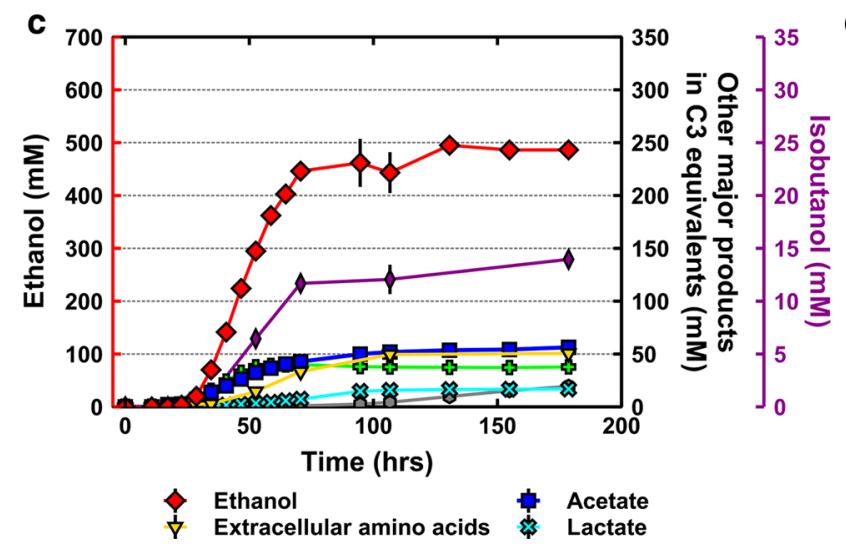

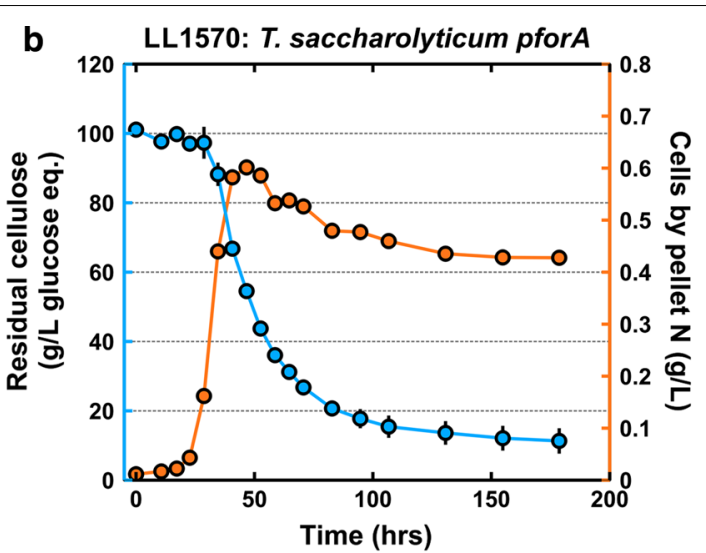

Cells by pellet $\mathrm{N}$ (g nitrogen/L)

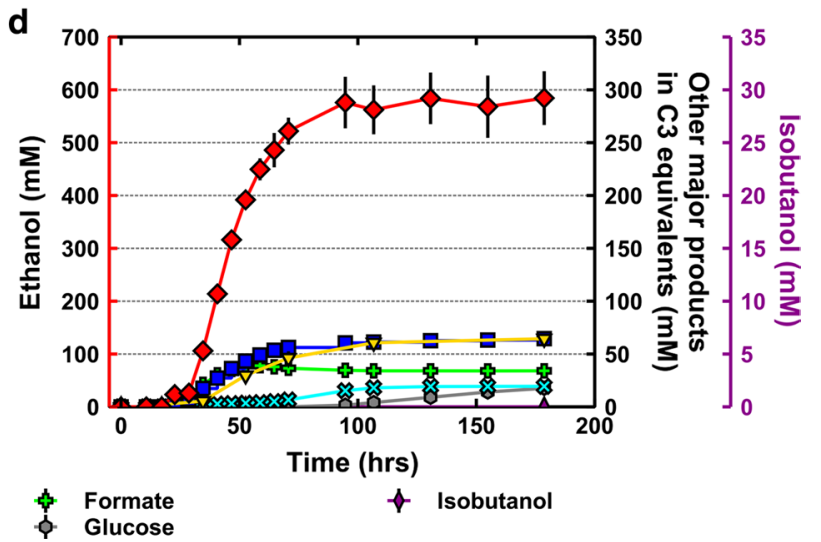

Glucose

Fig. 4 Comparison of the effect of fermentation with native C. thermocellum pfors (strain LL1319) or T. saccharolyticum pforA (strain LL1570). Substrate utilization as residual cellulose (light blue) and cells by pellet nitrogen (orange) versus fermentation times for strains LL1319 (a) and LL1570 (b). Major fermentation products—ethanol (red), acetate (dark blue), lactate (light blue), formate (light green), glucose (gray), isobutanol (purple), and extracellular amino acids (yellow) from the same fermentation runs for strains LL1319 (c) and LL1570 (d). Error bars represent mean absolute deviation ( $n=2$ fermentations). The data shown is for representative fermentations. Data for additional replicates is presented in Additional file 1:Table S5 and Figure S3. See Additional file 3 for tabular presentation of the data

Introducing just the T. saccharolyticum pforA did not improve ethanol titer, but it slightly shifted the ethanol to acetate ratio in favor of ethanol production. When the $T$. saccharolyticum pforA was expressed alongside the previously introduced T. saccharolyticum adhA, $n f n A B$, and $a d h E^{G 544 D}$ genes, ethanol production improved. These observations support the hypothesis that pforA is an important component of the T. saccharolyticum pyruvate-to-ethanol pathway. Furthermore, the pforA from T. saccharolyticum was able to functionally complement the deletion of the five annotated $C$. thermocellum pfor genes.

Isobutanol production in strain LL1570 was reduced below our limit of quantification $(0.1 \mathrm{mM})$. Fermentation data from a set of $C$. thermocellum strains with single deletions of each of the five annotated pfors points to pfor4 being responsible for isobutanol production. Given the interest in producing isobutanol either for use as a biofuel or as a feedstock chemical [36]; the knowledge that pfor4 is necessary for isobutanol production could be beneficial to further improve its production from cellulosic substrates.

The ability to use a T. saccharolyticum promoter to drive the expression of the pfor $A$-ferredoxin operon is relevant to future work involving gene expression in $C$. thermocellum. Whereas native $C$. thermocellum promoters have been characterized [31], with the enolase promoter being used successfully in this study to express T. saccharolyticum pforA, these promoters may still be subject to native transcriptional regulation, and therefore may not be suitable if constitutive gene expression is desired. It should be noted that other examples of heterologous promoters being used in C. thermocellum have been reported [19]. As heterologous gene expression becomes more prevalent in C. thermocellum [4, 22, 37], it will become increasingly necessary to develop libraries 
of non-native or synthetic genetic tools to avoid excessive duplicating of native DNA elements, which could contribute to genome instability.

Having now introduced six genes from the T. saccharolyticum pyruvate to ethanol pathway into C. thermocellum, we observe that there is still a $\sim 40 \mathrm{~g} / \mathrm{L}$ difference between the maximum ethanol titers achieved by engineered $C$. thermocellum $(25-30 \mathrm{~g} / \mathrm{L})$ [34] and those achieved by engineered $T$. saccharolyticum $(60-70 \mathrm{~g} / \mathrm{L})$ [5], suggesting that there remains more work to be done. With regard to the Pfor-catalyzed conversion of pyruvate to acetyl-CoA, one consideration is that the Pfor enzyme functions in tandem not only with ferredoxin, but also with ferredoxin: $\operatorname{NAD}(\mathrm{P})^{+}$oxidoreductase (Fnor). It is possible that titer limitations in engineered $C$. thermocellum are due to an un-optimized Pfor-ferredoxin-Fnor module. Some promising directions for further research include characterizing the Pfor-ferredoxin-Fnor modules of C. thermocellum and T. saccharolyticum in more detail, or engineering the module with better enzymes or through protein engineering of the existing enzymes to overcome possible substrate or cofactor inhibitions [38, 39].

\section{Additional files}

Additional file 1. Supplementary material for this study, including primer sequences (Table S1), promoter sequences (Table S2), fermentation data (Table S3, Table S4, Table S5, Figure S3 and Figure S4), chromosomal maps (Figure S1) and enzyme assay data (Figure S2).

Additional file 2. List of mutations observed among the strains described in this study

Additional file 3. Tabular presentation of the data from fermentation of high cellulose concentrations.

\section{Authors' contributions}

$\mathrm{SH}, \mathrm{EKH}, \mathrm{LT}, \mathrm{LRL}$, and DGO designed the experiments. SH performed plasmid construction, gene expression measurements, strain construction, biochemical assays, bottle and tube fermentations and subsequent sample analyses, and analyses of Sanger sequencing and whole genome resequencing results. PPL constructed strains LL1556, LL1559, LL1560, LL1563, and LL1564, and also measured isobutanol production from these five strains; JC performed biochemical assay measurements of these five strains. MIM performed genomic DNA extractions from C. thermocellum strains for resequencing. $\mathrm{SH}, \mathrm{EKH}$, and RSW performed the high solid fermentation experiments, as well as the sample analyses. SH and DGO wrote the manuscript. EKH, LRL, and DGO revised the manuscript. All authors read and approved the final manuscript.

\section{Author details}

1 Thayer School of Engineering, Dartmouth College, 14 Engineering Drive, Hanover, NH 03755, USA. ${ }^{2}$ Bioenergy Science Center, Oak Ridge National Laboratories, Oak Ridge, TN 37830, USA. ${ }^{3}$ Center for Bioenergy Innovation, Oak Ridge National Laboratories, Oak Ridge, TN 37830, USA. ${ }^{4}$ Department of Biological Sciences, Dartmouth College, Hanover, NH 03755, USA. ${ }^{5}$ University of California, Los Angeles, Los Angeles, CA 90095, USA.

\section{Acknowledgements}

We thank Janet W. Westpheling and Joseph Groom for the gift of plasmid pJGW37. We thank Johannes P. van Dijken for the useful feedback. The BioEnergy Science Center and Center for Bioenergy Innovation are US Department of Energy Bioenergy Research Centers supported by the Office of Biological and Environmental Research in the DOE Office of Science. Resequencing was performed by the Department of Energy Joint Genome Institute, a DOE Office of Science User Facility, and is supported by the Office of Science of the US Department of Energy under contract number DE-AC02-05CH11231.

\section{Competing interests}

Lee R. Lynd is a founder of the Enchi Corporation, which has a financial interest in Clostridium thermocellum.

\section{Availability of data and materials}

All data generated or analyzed during this study are included in the published article and its additional files. DNA sequences and resequencing results are available from GenBank via their accession numbers (see Table 2).

\section{Consent for publication \\ Not applicable.}

Ethics approval and consent to participate

Not applicable.

\section{Funding}

The BioEnergy Science Center and Center for Bioenergy Innovation are US Department of Energy Bioenergy Research Centers supported by the Office of Biological and Environmental Research in the DOE Office of Science. Resequencing was performed by the Department of Energy Joint Genome Institute, a DOE Office of Science User Facility, and is supported by the Office of Science of the US Department of Energy under contract number DE-AC02-05CH11231.

\section{Publisher's Note}

Springer Nature remains neutral with regard to jurisdictional claims in published maps and institutional affiliations.

Received: 5 July 2018 Accepted: 27 August 2018

Published online: 06 September 2018

\section{References}

1. Olson DG, McBride JE, Shaw AJ, Lynd LR. Recent progress in consolidated bioprocessing. Curr Opin Biotechnol. 2012;23:396-405. https://doi. org/10.1016/j.copbio.2011.11.026.

2. Lynd LR, Liang X, Biddy MJ, Allee A, Cai H, Foust T, et al. Cellulosic ethanol: status and innovation. Curr Opin Biotechnol. 2017:45:202-11. https://doi. org/10.1016/j.copbio.2017.03.008.

3. Dien BS, Cotta MA, Jeffries TW. Bacteria engineered for fuel ethanol production: current status. Appl Microbiol Biotechnol. 2003;63:258-66.

4. Hon S, Olson DG, Holwerda EK, Lanahan AA, Murphy SJ, Maloney MI, et al. The ethanol pathway from Thermoanaerobacterium saccharolyticum improves ethanol production in Clostridium thermocellum. Metab Eng. 2017:42:175-84. https://doi.org/10.1016/j.ymben.2017.06.011.

5. Herring CD, Kenealy WR, Shaw AJ, Covalla SF, Olson DG, Zhang J, et al. Strain and bioprocess improvement of a thermophilic anaerobe for the production of ethanol from wood. Biotechnol Biofuels. 2016;9:125. https ://doi.org/10.1186/s13068-016-0536-8.

6. Olson DG, Sparling R, Lynd LR. Ethanol production by engineered thermophiles. Curr Opin Biotechnol. 2015;33:130-41. https://doi. org/10.1016/j.copbio.2015.02.006.

7. Ma K, Hutchins A, Sung SJS, Adams MWW. Pyruvate ferredoxin oxidoreductase from the hyperthermophilic archaeon, Pyrococcus furiosus, functions as a CoA-dependent pyruvate decarboxylase. Proc Natl Acad Sci. 1997:94:9608-13. https://doi.org/10.1073/pnas.94.18.9608. 
8. Chabriere E, Cavazza C, Contreras-Martel C, Fontecilla-Camps JC. Pyruvate-ferredoxin oxidoreductase. Encycl Inorg Bioinorg Chem. 2011. https://doi.org/10.1002/9781119951438.eibc0647.

9. Townson SM, Upcroft A, Upcroft P. Characterisation and purification of pyruvate:ferredoxin oxidoreductase from Giarda duodenalis. Mol Biochem Parasitol. 1996;79:183-93.

10. Xiong W, Lin PP, Magnusson L, Warner L, Liao JC, Maness P-C, et al. $\mathrm{CO}_{2}$-fixing one-carbon metabolism in a cellulose-degrading bacterium Clostridium thermocellum. Proc Natl Acad Sci. 2016. https://doi. org/10.1073/pnas.1605482113.

11. Zhou J, Olson DG, Lanahan AA, Tian L, Murphy SJ-L, Lo J, et al. Physiological roles of pyruvate ferredoxin oxidoreductase and pyruvate formatelyase in Thermoanaerobacterium saccharolyticum JW/SL-YS485. Biotechnol Biofuels. 2015;8:138. https://doi.org/10.1186/s13068-015-0304-1.

12. Verbeke TJ, Zhang X, Henrissat B, Spicer V, RydzakT, Krokhin OV, et al. Genomic evaluation of Thermoanaerobacter spp. for the construction of designer co-cultures to improve lignocellulosic biofuel production. PLoS ONE. 2013:8:e59362. https://doi.org/10.1371/journal.pone.0059362.

13. Tian L, Lo J, Shao X, Zheng T, Olson DG, Lynd LR. Ferredoxin:NAD ${ }^{+}$ Oxidoreductase of Thermoanaerobacerium saccharolyticum and Its Role in Ethanol Formation. Appl Environ Microbiol. 2016;82:7134-41.

14. Lo J, Zheng T, Olson DG, Ruppertsberger N, Tripathi SA, Guss AM, et al. Deletion of $n f n A B$ in Thermoanaerobacterium saccharolyticum and its effect on metabolism. J Bacteriol. 2015;197:2920-9. https://doi. org/10.1128/JB.00347-15.

15. Zheng T, Olson DG, Murphy SJ-L, Shao X, Tian L, Lynd LR. Both adhE and a separate NADPH-dependent alcohol dehydrogenase, adhA, are necessary for high ethanol production in Thermoanaerobacterium saccharolyticum. $J$ Bacteriol. 2017;199:1-10. https://doi.org/10.1128/JB.00542-16.

16. Gibson DG. Enzymatic assembly of overlapping DNA fragments. Methods Enzymol. 2011;498:349-61. https://doi.org/10.1016/B978-0-12-38512 0-8.00015-2.

17. Olson DG, Lynd LR. Transformation of Clostridium thermocellum by electroporation. Methods in enzymology. 2012. https://doi.org/10.1016/ b978-0-12-415931-0.00017-3.

18. Guss AM, Olson DG, Caiazza NC, Lynd LR. Dcm methylation is detrimental to plasmid transformation in Clostridium thermocellum. Biotechnol Biofuels. 2012:5:30. https://doi.org/10.1186/1754-6834-5-30.

19. Groom J, Chung D, Olson DG, Lynd LR, Guss AM, Westpheling J. Promiscuous plasmid replication in thermophiles: use of a novel hyperthermophilic replicon for genetic manipulation of Clostridium thermocellum at its optimum growth temperature. Metab Eng Commun. 2016;3:30-8. https ://doi.org/10.1016/j.meteno.2016.01.004.

20. Olson DG, Lynd LR. Computational design and characterization of a temperature-sensitive plasmid replicon for gram positive thermophiles. J Biol Eng. 2012. https://doi.org/10.1186/1754-1611-6-5.

21. Livak KJ, Schmittgen TD. Analysis of relative gene expression data using real-time quantitative PCR and the $2^{-\triangle \Delta C T}$ method. Methods. 2001;25:402-8. https://doi.org/10.1006/meth.2001.1262.

22. Hon S, Lanahan AA, Tian L, Giannone RJ, Hettich RL, Olson DG, et al. Development of a plasmid-based expression system in Clostridium thermocellum and its use to screen heterologous expression of bifunctional alcohol dehydrogenases (adhEs). Metab Eng Commun. 2016. https://doi. org/10.1016/j.meteno.2016.04.001.

23. Lo J, Olson DG, Murphy SJ-L, Tian L, Hon S, Lanahan A, et al. Engineering electron metabolism to increase ethanol production in Clostridium thermocellum. Metab Eng. 2017;39:71-9. https://doi.org/10.1016/j.ymben 2016.10.018.

24. Espah Borujeni A, Channarasappa AS, Salis HM. Translation rate is controlled by coupled trade-offs between site accessibility, selective RNA unfolding and sliding at upstream standby sites. Nucleic Acids Res. 2014;42:2646-59.

25. Salis HM, Mirsky EA, Voigt CA. Automated design of synthetic ribosome binding sites to control protein expression. Nat Biotechnol. 2009;27:94650. https://doi.org/10.1038/nbt.1568.

26. Wang S, Huang H, Moll J, Thauer RK. NADP ${ }^{+}$reduction with reduced ferredoxin and $\mathrm{NADP}^{+}$reduction with $\mathrm{NADH}$ are coupled via an electron-bifurcating enzyme complex in Clostridium kluyveri. J Bacteriol. 2010;192:5115-23. https://doi.org/10.1128/JB.00612-10.

27. Holwerda EK, Thorne PG, Olson DG, Amador-Noguez D, Engle NL, Tschaplinski TJ, et al. The exometabolome of Clostridium thermocellum reveals overflow metabolism at high cellulose loading. Biotechnol Biofuels. 2014;7:155. https://doi.org/10.1186/s13068-014-0155-1.

28. Holwerda EK, Hirst KD, Lynd LR. A defined growth medium with very low background carbon for culturing Clostridium thermocellum. J Ind Microbiol Biotechnol. 2012;39:943-7.

29. Holwerda EK, Ellis LD, Lynd LR. Development and evaluation of methods to infer biosynthesis and substrate consumption in cultures of cellulolytic microorganisms. Biotechnol Bioeng. 2013;110:2380-8.

30. Richards FJ. A flexible growth function for emperical use. J Exp Bot. 1959;10:290-300. https://doi.org/10.1093/jxb/10.2.290.

31. Olson DG, Maloney M, Lanahan AA, Hon S, Hauser LJ, Lynd LR. Identifying promoters for gene expression in Clostridium thermocellum. Metab Eng Commun. 2015;2:23-9. https://doi.org/10.1016/j.meteno.2015.03.002.

32. Raman B, McKeown CK, Rodriquez M, Brown SD, Mielenz JR. Transcriptomic analysis of Clostridium thermocellum ATCC 27405 cellulose fermentation. BMC Microbiol. 2011;11:134. https://doi. org/10.1186/1471-2180-11-134.

33. Gowen CM, Fong SS. Genome-scale metabolic model integrated with RNAseq data to identify metabolic states of Clostridium thermocellum. Biotechnol J. 2010;5:759-67.

34. Tian L, Papanek B, Olson DG, Rydzak T, Holwerda EK, Zheng T, et al. Simultaneous achievement of high ethanol yield and titer in Clostridium thermocellum. Biotechnol Biofuels. 2016:9:1-11.

35. Huang $H$, Hu L, Yu W, Li H, Tao F, Xie H, et al. Heterologous overproduction of $2\left[4 \mathrm{Fe}_{4} \mathrm{~S}\right]$ - and $\left[2 \mathrm{Fe}_{2} \mathrm{~S}\right]$-type clostridial ferredoxins and $\left[2 \mathrm{Fe}_{2} \mathrm{~S}\right]$-type agrobacterial ferredoxin. Protein Expr Purif. 2016;121:1-8. https://doi. org/10.1016/j.pep.2015.12.019.

36. Lin PP, Mi L, Morioka AH, Yoshino KM, Konishi S, Xu SC, et al. Consolidated bioprocessing of cellulose to isobutanol using Clostridium thermocellum. Metab Eng. 2015;31:44-52. https://doi.org/10.1016/j.ymben.2015.07.001.

37. Tian L, Perot SJ, Hon S, Zhou J, Liang X, Bouvier JT, et al. Enhanced ethanol formation by Clostridium thermocellum via pyruvate decarboxylase. Microb Cell Fact. 2017;16:1-10.

38. Kim Y, Ingram LO, Shanmugam KT. Construction of an Escherichia coli K-12 mutant for homoethanologenic fermentation of glucose or xylose without foreign genes. Appl Environ Microbiol. 2007;73:1766-71.

39. Kim Y, Ingram LO, Shanmugam KT. Dihydrolipoamide dehydrogenase mutation alters the NADH sensitivity of pyruvate dehydrogenase complex of Escherichia coli K-12. J Bacteriol. 2008;190:3851-8. 